\title{
Cybersickness Analysis Using Symbolic Machine Learning Algorithms
}

\author{
Thiago Porcino \\ Universidade Federal Fluminense \\ Niterói, Brazil \\ tmalheiros@id.uff.br
}

\author{
Daniela Trevisan \\ Universidade Federal Fluminense \\ Niterói, Brazil \\ daniela@ic.uff.br
}

\author{
Esteban Clua \\ Universidade Federal Fluminense \\ Niterói, Brazil \\ esteban@ic.uff.br
}

\begin{abstract}
Virtual reality (VR) and head-mounted displays are constantly gaining popularity in various fields such as education, military, entertainment, and health. Although such technologies provide a high sense of immersion, they can also trigger symptoms of discomfort. This condition is called cybersickness (CS) and is quite popular in recent VR publications. This work proposes a novel experimental analysis using symbolic machine learning to rank potential causes of CS in VR games. We estimate CS causes and rank them according to their impact on the classical machine learning classification task. Experiments are performed using two VR games and 6 experimental protocols along with 37 valid samples from a total of 88 volunteers.
\end{abstract}

\section{CCS CONCEPTS}

- Human-centered computing $\rightarrow$ Virtual reality; • Theory of computation $\rightarrow$ Machine learning theory.

\section{KEYWORDS}

virtual reality, head-mounted displays, cybersickness, games, machine learning

\section{ACM Reference Format:}

Thiago Porcino, Daniela Trevisan, and Esteban Clua. 2021. Cybersickness Analysis Using Symbolic Machine Learning Algorithms. In Proceedings of SVR '21: Workshop on Thesis and Dissertations (SVR '21). SOL, Porto Alegre, RS, Brazil, 2 pages.

\section{INTRODUCTION}

Activities such as virtual training environments, simulations and entertainment in immersive virtual formats are constantly becoming more popular with the continued development and public interest in VR technologies over the last years. Head-mounted displays (HMDs) is one of the means of achieving immersive VR. HMDs are used for various purposes in the industry such as in games that focus on entertainment, military, education, therapy, and simulators for numerous contexts.

Unfortunately, HMDs are strongly related to frequent manifestations of discomfort. Among the possible manifestations, cybersickness (CS) deserves special attention as it is the most frequent and is usually associated to long exposures to HMDs. These symptoms of discomfort impact the user experience and affects the profit and coverage of the VR game industry. This work is related to machine learning for VR/AR/MR SVR topic and doctoral thesis category.

SVR '21, 18th-21st October, 2021, Gramado, RS

\section{CONTRIBUTIONS}

Through this research, we have been contributing to mitigate cybersickness problems in VR environments:

Providing an extensive cybersickness literature review. Our review facilitates researchers to identify the leading causes for most discomfort situations in VR environments and associate the most recommended strategies to minimize such discomfort. Additionally, Kemeny et al. [1] cited part of this work in their book. At the moment, more than 80 works cited us [9].

SVR' Best Review Paper Award. Part of this work [7] was awarded at the symposium on virtual and augmented reality (SVR). Additionally, we submitted an extended version of this study to SBC' Journal on Interactive Systems [4].

Proposing the cybersickness profile questionnaire. We create the cybersickness profile questionnaire (CSPQ) based on our literature findings. The CSPQ contains 9 questions about user profile tied to cybersickness manifestations [6].

Proposing symbolic machine learning models to identify causes of cybersickness in VR environments. We are the first to use symbolic classifiers (decision tree and random forest) to estimate CS causes during a gameplay experience [3]. Additionally, we submitted an extended version of this study to Entertainment Computing Journal [5].

Proposing an experimental methodology to capture user and gameplay data tied to cybersickness. We created and conducted an iterative evaluating protocol methodology and proposed two VR games (a racing game and a flight game) for user and gameplay data acquisition [8].

Providing a public VR users database. The raw dataset of this work is published in a public domain for further reproduction and comparisons [2].

\section{PROPOSED SOLUTION}

We propose an approach that enables cause estimation while the user is under the VR condition. Our solution consists of following the prediction course through symbolic machine learning algorithms (decision tree and random forest) while using feature frequency and the node height to estimate the influence of the attributes.

The logical prediction path of the decision trees inherits a personal fingerprint associated to attribute weights. Usually, attributes that are closer to the tree root are more important, as they often reduce the chaos in data more than the rest (information gain, less entropy). As a general rule, the frequency in which attributes appear in the decision path is also an important piece of information. We combine these two aspects to estimate the importance of the attribute (i.e., the most important causes of discomfort). 
Let us suppose a decision tree described by 9 decision nodes, as shown in Figure 1, which contains in their conditions the features G (gender), R (rotation Z), A (acceleration) and S (speed). Furthermore, let us consider a path for an instance that was predicted as discomfort, highlighted in green in the same figure.

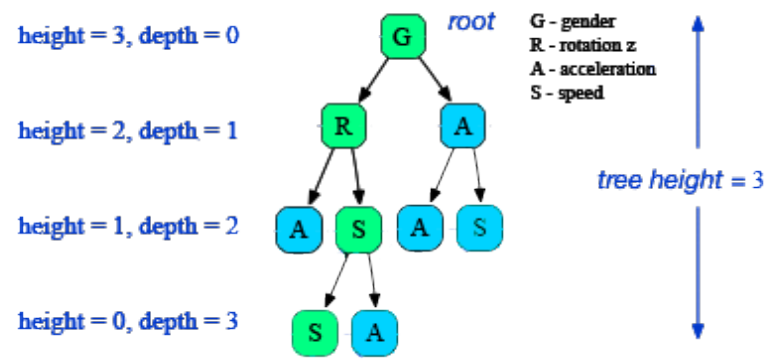

Figure 1: A decision tree model. The green path illustrates the decision path that uses the attributes $G, R, S$, and $S$.

We compute a potential-cause score (PCS) by summing up the heights of these features in the green path. In this case, $G$ appeared once and has height 3 , rotation $\mathrm{R}$ has height 2 and speed $\mathrm{S}$ has heights 1 and 0 . Next, the output is divided by the sum of all depths of the tree, as follows: $\mathrm{G}=3 / 6$ or $0.5, \mathrm{R}=2 / 6$ or approximately 0.33 and $S=1 / 6$ or approximately 0.17 . In this case, we estimate gender as the most relevant cause for CS (Equation 1).

$$
\operatorname{PCS}(F)=\frac{\sum_{h=0}^{\text {max height }}\left\{\begin{array}{c}
h, \text { if } F \text { belongs to the height } \\
0, \text { otherwise }
\end{array}\right.}{\sum_{h=0}^{\max \text { height }} h}
$$

In equation $1, h$ varies from 0 to the maximal tree height, and $F$ is the feature being evaluated. PCS is computed considering just the decision path (e.g., the one highlighted in green)

Furthermore, the random forest model can be considered a set of decision trees. We sum the PCS results from each tree $t$ if this tree's final decision is equal to the RF final decision. Otherwise, we sum 0 in this iteration. In Equation 2, we sum the PCS results from each tree $t$ if the classification result of $t$ is equal to the final RF classification result, where votes from several trees are scored together and a single class, e.g., the mode, is chosen as the classification result. Otherwise, we sum 0 at this iteration.

$$
P C S_{R F}(F)=\sum_{t \in T}\left\{\begin{array}{c}
P C S(F), \text { if tree } t \text { decision }=R F \text { final decision } \\
0, \text { otherwise }
\end{array}\right.
$$

\section{RESULTS}

Our results (Figure 2) show that rotation and acceleration triggered cybersickness more frequently in a flight game in contrast to a race game. We could also observe that participants that are less experienced with VR are more prone to feel discomfort. Former experience plays a more important role on the race game, as this game provides more liberty to the user in terms of controllers, more displacement alternatives and a more self-controlled acceleration. Additionally, time is a crucial variable is terms of CS mitigation. Conclusively, different causes that trigger discomfort arise based on short or long term VR exposures. As final remark, we suggest strategies for mitigating $\mathrm{CS}$ for these two scenarios.

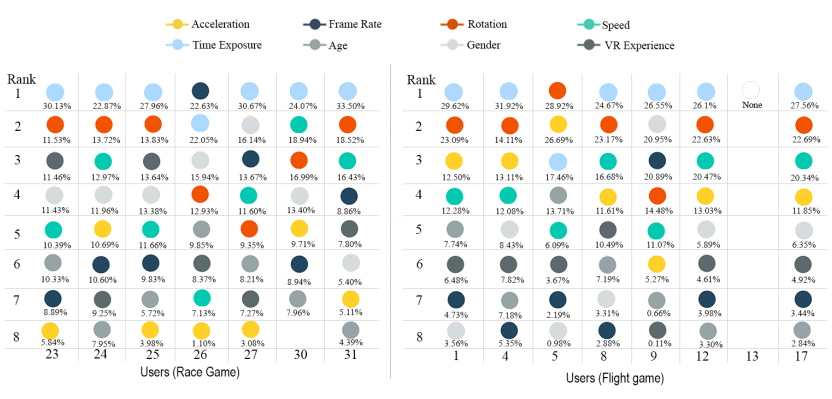

Figure 2: Random Forest feature ranking for the race and flight game.

\section{CONCLUSIONS}

The human-readable characteristic of decision tree and random forest helped us to understand the discomfort manifestation reasons in two different games. This fact, show that symbolic machine learning models are a good way to identify CS causes, where understability is essential to highlight causes and suggest strategies. Designers can benefit from our approach by enabling the selection of the best strategy for a specific context.

\section{REFERENCES}

[1] Andras Kemeny, Jean-Rémy Chardonnet, and Florent Colombet. 2020. Getting Rid of Cybersickness: In Virtual Reality, Augmented Reality, and Simulators. Springer Nature.

[2] Thiago Porcino. [n.d.]. Cybersickness Dataset. https://github.com/tmp1986/ UFFCSData. Accessed: 2021-04-03.

[3] Thiago Porcino, Flavia Bernardini, Erick O Rodrigues, Alexandre Silva, Esteban Clua, and Daniela Trevisan. 2021. A symbolic machine learning approach for cybersickness potential-cause estimation. In International Conference on Entertainment Computing. Springer [Accepted].

[4] Thiago Porcino, Erick O Rodrigues, Flavia Bernardini, Daniela Trevisan, and Esteban Clua. 2021. A cybersickness review: causes, strategies, and classification methods. SBC fournal on Interactive Systems [Submitted] (2021).

[5] Thiago Porcino, Erick O Rodrigues, Flavia Bernardini, Daniela Trevisan, and Esteban Clua. 2021. Identifying Cybersickness Causes in Virtual Reality Games Using Symbolic Machine Learning Algorithms. Entertainment Computing [Submitted] (2021).

[6] Thiago Porcino, Erick O Rodrigues, Alexandre Silva, Esteban Clua, and Daniela Trevisan. 2020. Using the gameplay and user data to predict and identify causes of cybersickness manifestation in virtual reality games. In 2020 IEEE 8th International Conference on Serious Games and Applications for Health (SeGAH). IEEE, 1-8.

[7] Thiago Porcino, Daniela Trevisan, and Esteban Clua. 2020. Minimizing cybersickness in head-mounted display systems: causes and strategies review. In 2020 22nd Symposium on Virtual and Augmented Reality (SVR). IEEE, 154-163.

[8] Thiago Porcino, Daniela Trevisan, and Esteban Clua. 2021. An experimental methodology to capture user and gameplay data tied to cybersickness. In Proceedings of the 1st XR in Games Workshop. SBC.

[9] Thiago M Porcino, Esteban Clua, Daniela Trevisan, Cristina N Vasconcelos, and Luis Valente. 2017. Minimizing cyber sickness in head mounted display systems: design guidelines and applications. In Serious Games and Applications for Health (SeGAH), 2017 IEEE 5th International Conference on. IEEE, 1-6. 